\title{
SOFT X-RAY SPECTROMICROSCOPY AND ITS APPLICATION TO SEMICONDUCTOR MICROSTRUCTURE CHARACTERIZATION
}

\author{
F. Gozzo, K. Franck, M.R. Howells, Z. Hussain, A. Warwick, \\ H.A. PADMORE
}

Advanced Light Source, Lawrence Berkeley National Laboratory

1 Cyclotron Rd., Berkeley, CA 94720, USA

and B.B. TripletT

Intel Corporation, 3065 Bowers Ave., Santa Clara, CA 95052, USA

\begin{abstract}
The universal trend towards device miniaturization has driven the semiconductor industry to develop sophisticated and complex instrumentation for the characterization of microstructures. Many significant problems of relevance to the semiconductor industry cannot be solved with conventional analysis techniques, but can be addressed with soft X-ray spectromicroscopy. An active spectromicroscopy program is being developed at the Advanced Light Source, attracting both the semiconductor industry and the materials science academic community. Examples of spectromicroscopy techniques are presented. An Advanced Light Source $\mu$-XPS spectromicroscopy project is discussed, involving the first microscope completely dedicated and designed for microstructure analysis on patterned silicon wafers.
\end{abstract}

PACS numbers: 79.60.-i, 61.16.-d, 85.40.-e

\section{Multilevel metallization technology: an overview}

With the term metallization we refer to the electrical connection of microchip devices between themselves and to the external world [1]. We concentrate on metallization, recognized as one of the keys to success of continued miniaturization of devices and circuits, in relation to problems which can be addressed with soft $X$-ray spectromicroscopy.

Figure 1 is a scanning electron microscope (SEM) cross-section of a 4-level metal microchip with a minimum feature size of $0.35 \mu \mathrm{m}$. The wiring architecture is extremely complicated and develops in both horizontal and vertical directions. Reaching this level of complexity has required an enormous effort. Scaling towards smaller features continues and there are many challenging problems which need to be solved in order to maintain this scaling track. Decreasing dimensions have 


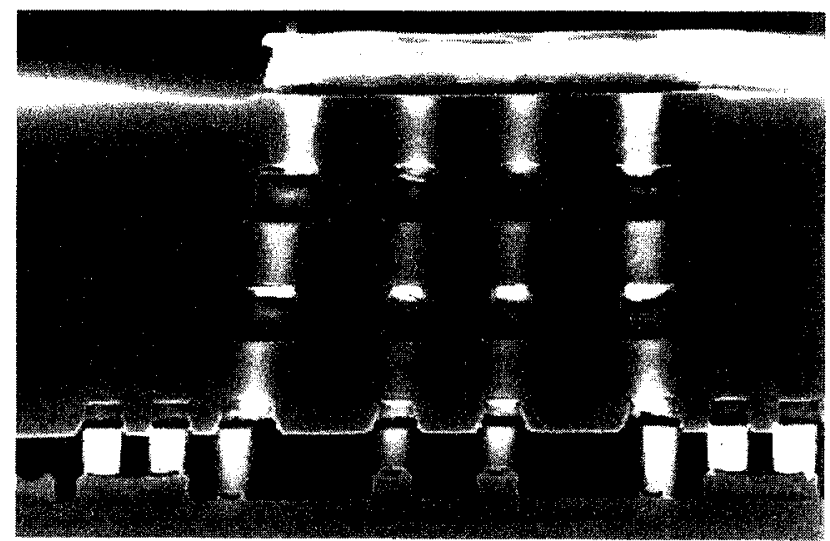

Fig. 1. SEM cross-section of a 5-level metallization structure, cross-section showing 4-layer metal, $0.35 \mu \mathrm{m}$ minimum feature size (courtesy of Intel).



(a)

Si p-type substrate

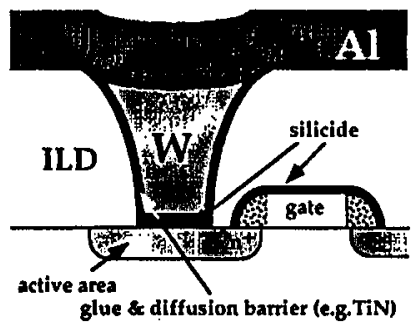

(b) Si p-type substrate

Fig. 2. Contact region cross-section: (a) tapered and (b) straight wall contact.

caused serious concern regarding electromigration, stress-voiding, adhesion failure, corrosion, and interdiffusion. These are some of the key fundamental issues of the reliability of metallization [1].

Figure 2 shows the transition through $1.0 \mu \mathrm{m}$ minimum feature size technology. Above $1 \mu \mathrm{m}$, the metal (typically aluminum) was physical vapor deposition (PVD) deposited on the transistor active region. As devices scaled below $1 \mu \mathrm{m}$, tapered contacts and vias* designs occupied too much space and, as the transistor $p-n$ junctions became smaller, the Al migration into the Si substrate induced junction spikings [1]. Tapered contacts and vias design was, then, replaced by straight wall contact and via filling. Aluminum-PVD was replaced by tungsten-chemical vapor deposition (CVD) technology for local interconnections (Fig. 2b) or high temperature Al reflow technology [2]. Diffusion barriers (Ti, TiN or TiW) were added to stop problems such as the diffusion of $\mathrm{Al}$ into $\mathrm{Si}$ during and after the Al reflow processing and $W_{6}$ attack during $W$ plug filling. Diffusion barriers

* Via - vertical opening used to connect on various layers of a device to one another and to the semiconducting substrate. 
were also found to provide better adhesion and mechanical contact. The tungsten contact resistivity in $\mathrm{W}$ plug filling was decreased by adding a silicide layer.

In the transition between 0.8 and $0.35 \mu \mathrm{m}, \mathrm{Al}$ was replaced by Al-alloys for higher reliability against electromigration [3] and via etch depth control required the use of etch stops.

For the $0.25 \mu \mathrm{m}$ and especially $0.18 \mu \mathrm{m}$ generation and below, the problems which today make the processes extremely complicated and expensive, are expected to become true limitations. Low contact resistivity and shorter delay time requirements for faster transistors, demand the integration of new low resistivity and/or low dielectric constant materials [4].

Soft X-ray spectromicroscopy is expected to have a big impact on problems which require chemical analyses on a microscopic scale. Although presently used microscopes offer very high spatial resolution (e.g. transmission electron microscope, TEM) or elemental analysis (e.g. SEM, time of flight-secondary ions mass spectroscopy, TOF-SIMS) many critical problems require knowledge of the chemistry of materials in the surface or near surface regions. Examples are adhesion and corrosion where the physical processes are related to the interface chemical structure. Soft X-ray spectromicroscopy will have a dramatic effect on this kind of problems by combining traditional surface science techniques with microscopic capabilities made possible by ultrahigh brightness third generation soft X-ray synchrotron radiation sources. Size related problems were observed in via and contact etching and filling, as well as in the diffusion barrier properties. Soft X-ray spectromicroscopy will allow a non-destructive analysis of multiple interfaces and give chemical composition information on microscopic scale.

\section{Soft X-ray spectromicroscopy}

With the term spectromicroscopy we refer to all the experimental techniques which combine conventional spectroscopy methods with high lateral resolution microscopy [5]. Spectromicroscopy experiments produce two-dimensional micrographs and/or spectra over microscopic areas. Images provide chemical and topographical information if a contrast mechanism is present.

$\mathrm{X}$-ray spectromicroscopy can operate in scanning and imaging modes. Figure 3 and Table show a schematic representation of these two modes and examples of the existing spectromicroscopes [6-16].

In the scanning mode, micrographs are obtained by scanning a focused monochromatic photon beam on the sample surface, while collecting the electrons or photons emitted by the sample. The spatial resolution then, depends on the specific characteristic of the focused photon beam [16, 17]. A compromise must be reached between the size of the X-ray spot on the sample surface and the photon beam intensity necessary to obtain both an acceptable signal-to-noise ratio and the high energy resolution required for spectroscopy. The recent development of new ultrahigh brightness synchrotron radiation sources like the Advanced Light Source (ALS) in Berkeley and Elettra in Trieste, has made spectromicroscopy a practical technology.

In the imaging mode of operation, micrographs are obtained by using electron or photon optics. The sample is flooded with $\mathrm{X}$-rays and the resolution of the 


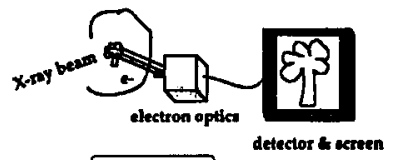

(a) imaging

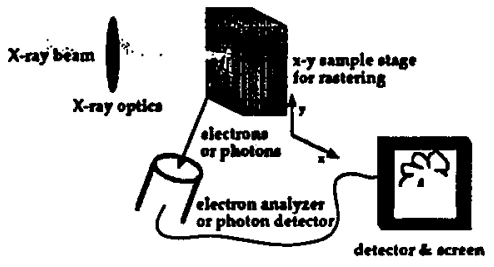

(c)
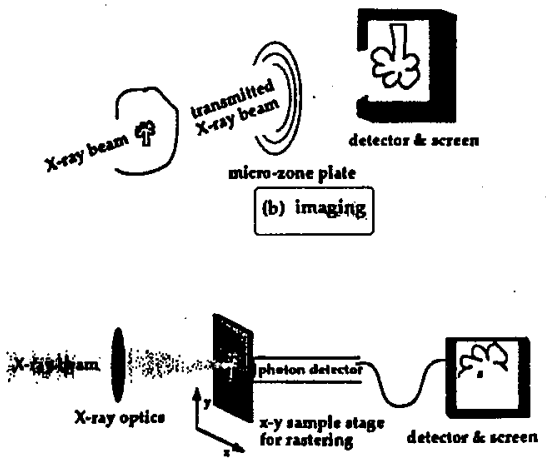

(d)

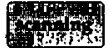

Fig. 3. Imaging and scanning modes of operation (compare with Table).

TABLE

The imaging and scanning modes of operation with examples on existing spectromicroscopes.

\begin{tabular}{|c|c|c|c|}
\hline \multirow{3}{*}{ Imaging } & \multirow[t]{2}{*}{ Electron optics } & Photoemission & $\begin{array}{l}\text { photon in } \rightarrow \text { electron out } \\
\text { (e.g. SCIENTA } 300[6] \text {, } \\
\text { ESCASCOPE [7]) }\end{array}$ \\
\hline & & $\begin{array}{l}\text { Absorption near } \\
\text { an edge }\end{array}$ & $\begin{array}{l}\text { photon in } \rightarrow \text { electron out } \\
\text { (e.g. PEEM [8], PRISM [9]) }\end{array}$ \\
\hline & X-rays optics & Transmission & $\begin{array}{l}\text { photon in } \rightarrow \text { photon out } \\
\text { (e.g. XM [10], XM-1 [11]) }\end{array}$ \\
\hline \multirow{4}{*}{ Scanning } & \multirow{4}{*}{$\mathrm{X}$-rays optics } & Photoemission & $\begin{array}{l}\text { photon in } \rightarrow \text { electron out } \\
\text { (e.g. MAXIMUM [12], SPEM [13] } \\
\text { K\&B } \mu \text {-XPS, ultra-ESCA [14]) }\end{array}$ \\
\hline & & $\begin{array}{l}\text { Absorption near } \\
\text { an edge }\end{array}$ & $\begin{array}{l}\text { photon in } \rightarrow \text { electron out } \\
\text { (e.g. ultra-ESCA [14]) }\end{array}$ \\
\hline & & Transmission & $\begin{array}{l}\text { photon in } \rightarrow \text { photon out } \\
\text { (e.g. STXM [15]) }\end{array}$ \\
\hline & & Fluorescence & $\begin{array}{l}\text { photon in } \rightarrow \text { photon out } \\
\text { (e.g. microprobe [16]) }\end{array}$ \\
\hline
\end{tabular}

image is limited by the aberrations of the optical systems. We focus on soft X-ray scanning photoemission spectromicroscopes, based on the detection and study of photoelectrons whose emission is stimulated by soft X-rays. In particular, we de- 
scribe here the MAXIMUM, SPEM, and KB $\mu$-XPSM spectromicroscopes, and present some results.

\subsection{Multiple Application X-Ray Imaging Undulator Microscope (MAXIMUM)}

MAXIMUM is a scanning soft $\mathrm{X}$-ray photoemission spectromicroscope which focuses undulator radiation using a multilayer coated Schwarzschild objective (SO) [18]. The image is formed by scanning the sample at the focal point and collecting photoelectrons. The microscope has been developed by Cerrina and his group in Wisconsin and has been recently moved at the ALS. The use of the ALS ultrahigh brightness source is expected to improve the microscope performances to the theoretical resolution limit [12].

The SO has the advantage of a relatively large numerical aperture $(\approx 0.2)$ and the opposite curvatures of the surfaces assign opposite signs to the geometrical aberrations of the two mirrors. Multilayer coatings are needed for reflectivity enhancement at normal incidence. These limit the operation of the microscope to a fixed photon energy. The present system at ALS operates at $130 \mathrm{eV}$. MAXIMUM's upgraded version, at Elettra in Trieste, overcomes this limitation by using a multiple objective system [19]. However, a photon energy upper limit of $250 \mathrm{eV}$, determined by the available multilayer coating materials, limits its application to the shallow core levels and valence band spectroscopy. Figure 4 shows a layout of MAXIMUM [12].

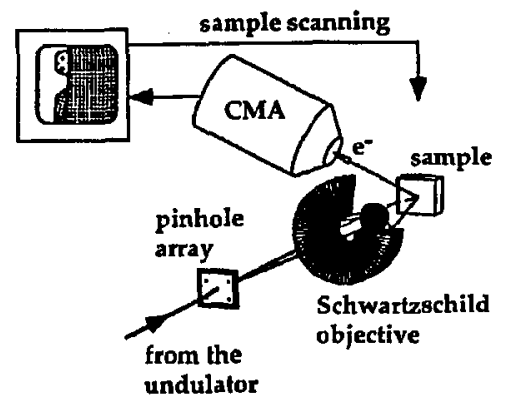

Fig. 4. Layout of MAXIMUM microscope (courtesy of Waiman $\mathrm{Ng}$ ).

One of the first applications of MAXIMUM to the study of semiconductor surfaces and interfaces was the search for possible lateral variations of semiconductor interface parameters in both metal/semiconductor and semiconductor/semiconductor interfaces [20,21]. Lateral variations of approximately $0.1 \mathrm{eV}$ in band bending were found by Gozzo et al. in a $\mathrm{GaSe} / \mathrm{Au}$ interface [20]. For a $\mathrm{GaSe} / \mathrm{Ge}$ interface, the observed lateral variations of the band lineup were approximately $0.4 \mathrm{eV}[21]$.

The image shown in Fig. 5 was obtained by focusing the photon beam on a cleaved GaSe surface covered with a 0.5 monolayer of $\mathrm{Ge}$ and scanning the sample while detecting the photoemission signal from the Ge $3 d$ states [21]. The image revealed substantial inhomogeneities in the signal, and in particular a line-shaped 


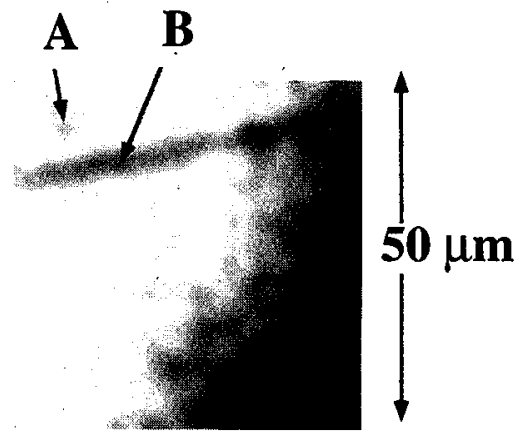

GaSe + 0.5 ML Ge

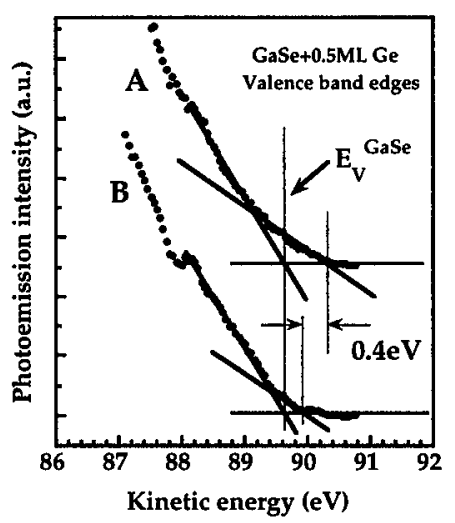

Fig. 5. (a) Scanning photoemission micrograph of a cleaved $50 \times 50 \mu \mathrm{m}^{2}$ GaSe surface with a $0.5 \mathrm{ML}$ Ge overlayer, taken at $61.2 \mathrm{eV}$ kinetic energy; (b) valence-band edge spectra; the clean substrate edge coincides for regions $A$ and $B$, whereas the Ge-derived edges of the curves differ by approximately $0.4 \mathrm{eV}$.

feature (region $B$ ) in the top portion of the analyzed area of the sample. The observed relative shift of the substrate and overlayer core levels in the two regions $A$ and $B$ (not shown here), indicated a rigid shift of the entire GaSe band structure and suggested a change from point $A$ to point $B$ in the energy lineup of the GaSe and $\mathrm{Ge}$ electronic states. The analysis of the valence electronic states in $A$ and $B$ near the edge (see Fig. 5) indeed showed that the valence band discontinuity $\Delta E_{\mathrm{v}}$ changed from place to place by approximately $0.4 \mathrm{eV}$, well beyond the experimental uncertainty.

\subsection{Scanning photoemission electron microscope (SPEM)}

In the scanning photoemission microscope the focusing optical system is a Fresnel zone plate (FZP) [22]. FZPs are diffractive elements made up of concentric circular zones of constant area. Compared to Schwarzschild objectives, they have a wider wavelength operation range, but the focal length is dependent on the wavelength and the numerical aperture is smaller. However, they are capable of focusing high energy $\mathrm{X}$-rays, allowing access to deeper core levels.

A new project involving the construction of two scanning zone plate microscopes, is now under development on beam line 7.0 at the ALS [23]. Figure 6 shows the microscopes layout. One of the microscopes is a UHV-SPEM. The second is a scanning transmission X-ray microscope (STXM) with photon detection either downstream of the sample or with detection of front surface electron yield. The ALS-SPEM is designed to form images by scanning the zone plate and not the sample [13-15] thus allowing heating and cooling systems to be attached to the stationary sample. The system will be commissioned in August 1996.

\subsection{Kirkpatrick-Baez $\mu$-X-ray photoemission spectromicroscope (KB $\mu$-XPSM)}

The Kirkpatrick-Baez $\mu$-X-ray photoemission spectromicroscope is a scanning soft $\mathrm{X}$-ray photoemission microscope which focuses bending magnet radiation 


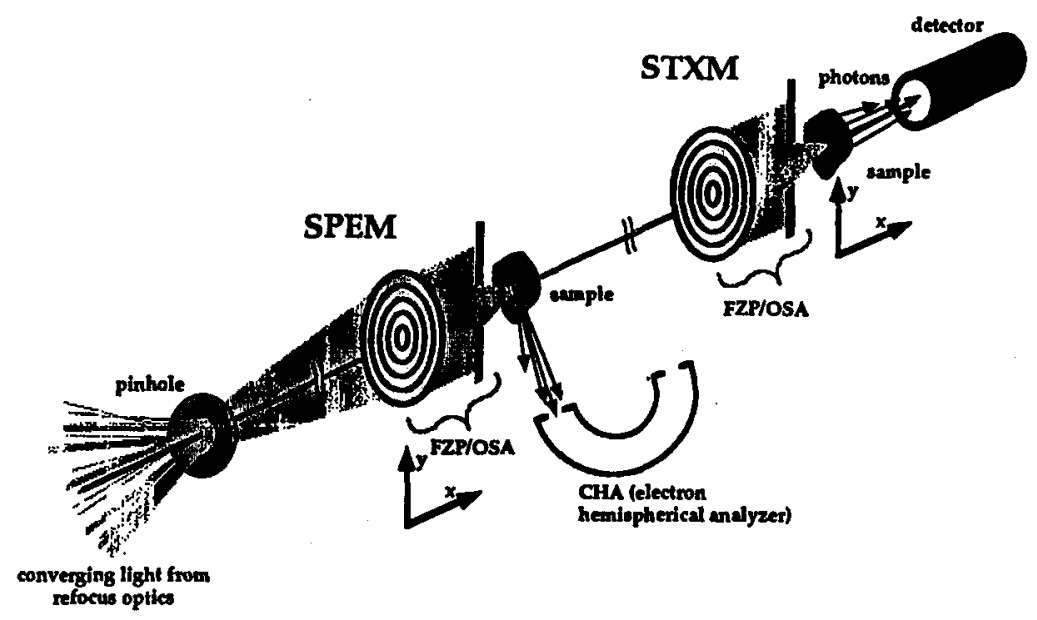

Fig. 6. ALS-SPEM/STXM microscopes layout.

using a pair of concave mirrors mounted in a Kirkpatrick-Baez configuration [24]. Operating at 30:1 demagnification, this optical system is designed to refocus the $\mathrm{X}$-ray beam from a $30 \mu \mathrm{m}$ to a $1 \mu \mathrm{m}$ image. The estimated flux in the microfocus is better than $10^{10} \mathrm{ph} / \mathrm{s}$. The KB $\mu$-XPSM microscope is part of a new ALS spectromicroscopy project which directly involves the semiconductor manufacturing industry. Figure 7 shows a schematic layout of the new ALS beam line designed to house the KB microscope and a planned upgraded version of a photoelectron emission microscope (PEEM2).

The originality of the KB $\mu$-XPSM project is not in its ultimate spatial resolution, but in the ability to combine spectromicroscopy capabilities with semiconductor industry requirements:

- Capability to quickly and accurately navigate over large silicon wafers and analyze specific regions previously identified with respect to fiducial reference points. (The present project will be able to handle 2 inches sections of silicon wafers, but is designed to scale to stages for full 8 inches wafers.)

- Large beam time allocation satisfied by the construction of a dedicated bending magnet beam line. A bending magnet, instead of an undulator beam line, sensibly limits the cost of the entire project, while providing the necessary photon flux for $1 \mu \mathrm{m}$ resolution experiments.

- Compatibility with the equipment required for wafer analysis (e.g. heating and cooling system, depth profiling, SEM detector, flood gun).

- User-friendly character of the entire system for a prompt support to development and fabrication tasks.

The inset in Fig. 7 shows the principle of operation of the KB microscope $[16,25]$. A simple spherical grating in a spherical grating monochromator (SGM) geometry is needed to monochromatize light for both the PEEM and the KB $\mu$-XPSM lines. $0.2 \mathrm{mrad}$ at the horizontal radiation fan is split off from the main 


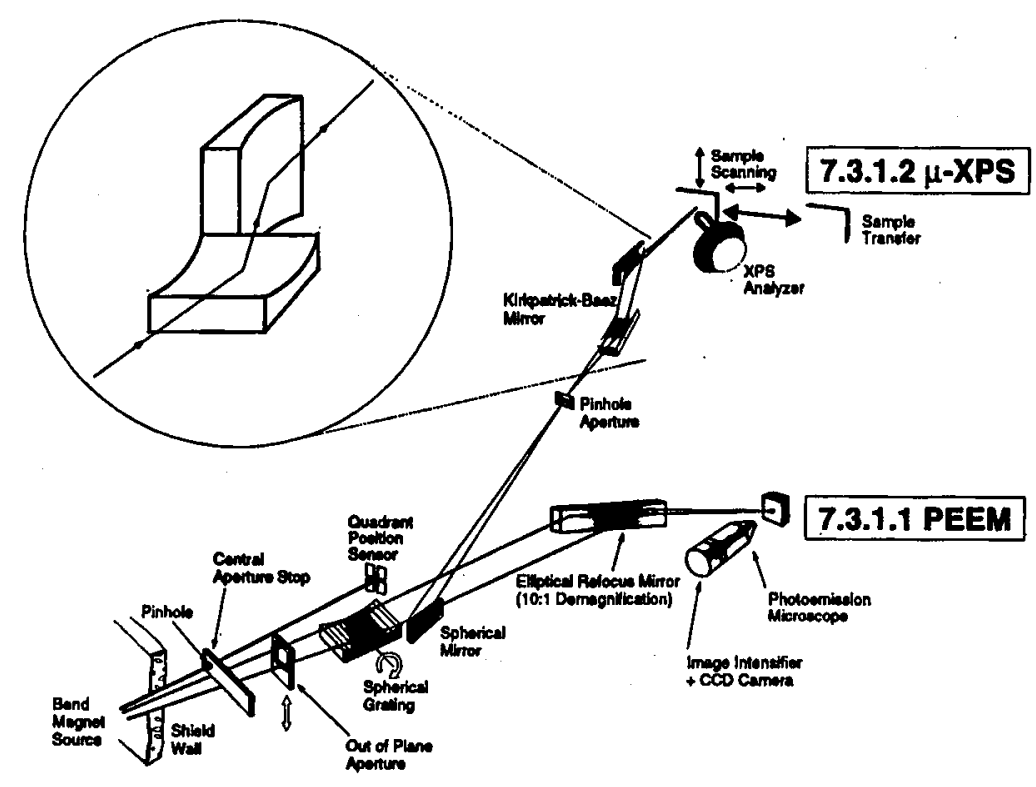

Fig. 7. Layout of the KB $\mu$-XPSM and PEEM2 beam line and microscopes.

beam by a spherical mirror and focused to a pinhole. This $20 \times 40 \mu \mathrm{m}$ pinhole provides the source of illumination for the following Kirkpatrick-Baez $(\mathrm{K}-\mathrm{B})$ mirror system. This consists of two crossed elliptical cylindrical mirrors operating at 20:1 and 40:1 demagnification factor. The system is designed to collect the maximum aperture, commensurate with obtaining good reflectivity from the mirrors [25]. The photon energy range is $250-1300 \mathrm{eV}$, with an expected spatial resolution of $1 \mu \mathrm{m}$. The system will be commissioned in October 1996.

\section{Conclusions}

Photoemission spectromicroscopy has already produced remarkable results in the study of fundamental semiconductor interface problems. The challenge to the synchrotron radiation community is, now, to use the available spectromicroscopy techniques to solve problems of real significance to the semiconductor manufacturing industry. This requires not only spatial resolution capabilities, but also the integration of existing standard industry techniques in an efficient and user friendly environment.

\section{Acknowledgments}

One of us, F.G., would like to acknowledge the Swiss National Science Foundation for supporting her grant in the United States. This work was partially supported by the Director, Office of Energy Research, Office of Basic Energy Sciences, Materials Sciences Division of the U.S. Department of Energy, under Contract No. DE-AC03-76SF00098. We thank Intel's Materials Technology and Components Research groups for characterizing samples used in the demonstration of the feasibility of X-ray spectromicroscopy applications. 


\section{References}

[1] S.P. Murarka, Metallization: Theory and Practice for VLSI and ULSI, Butterworth-Heinemann, Boston 1993.

[2] K. Kikuta, MRS Bull. XX, 53 (1995).

[3] Y. Shacham-Diamand, MRS Bull. XX, 78 (1995).

[4] P. Singer, Semicond. Int., 88 (1996).

[5] G. Margaritondo, F. Cerrina, Nucl. Instrum. Methods Phys. Res. A 291, 26 (1990).

[6] U. Gelius, B. Wannberg, P. Baltzer, H. Fellner-Feldegg, G. Carlson, C.G. Johansson, J. Larsson, P. Münger, G. Vegerfors, J. Electron Spectrosc. Relat. Phenom. 52, 747 (1990).

[7] P. Coxon, J. Krizek, M. Humpherson, I.R.M. Waedell, J. Electron. Spectrosc. Relat. Phenom. 52, 821 (1990).

[8] W. Engel, M.E. Kordesch, H.H. Rotermund, S. Kubala, A. von Oertzen, Ultramicroscopy 36, 148 (1991).

[9] D. Dunham, D.M. Desloge, G.F. Rempfer, W.P. Skoczylas, B.P. Tonner, Nucl. Instrum. Methods Phys. Res. A 347, 441 (1994).

[10] B. Niemann, D. Rudolph, G. Schmahl, Nucl. Instrum. Methods 208, 367 (1983).

[11] W. Meyer-Ilse, H. Medecki, L. Jochum, E. Anderson, D. Attwood, C. Magowan, R. Balhorn, M. Moronne, Synchr. Rad. News 8, 29 (1995).

[12] F. Cerrina, J. Electron Spectrosc. Relat. Phenom. 76, 9 (1995).

[13] H. Ade, J. Kirz, S. Hulbert, E. Johnson, E. Anderson, D. Kern, Nucl. Instrum. Methods Phys. Res. A 291, 126 (1990).

[14] J.D. Denlinger, E. Rotenberg, T. Warwick, G. Visser, J. Nordgren, J.H. Guo, P. Skytt, S.D. Kevan, K.S. McKutcheon, D. Shuh, J. Bucher, N. Edelstein, J.G. Tobin, B.P. Tonner, Rev. Sci. Instrum. 66, 1342 (1995).

[15] H. Rarbach, D. Shu, S.C. Feng, H. Ade, J. Kirz, I. McNulty, D.P. Kern, T.H.P. Chang, Y. Vladimirsky, N. Iskander, D. Attwood, K. McQuaid, S. Rothman, Rev. Sci. Instrum. 59, 52 (1988).

[16] M.R. Howells, J.B. Hastings, Nucl. Instrum. Methods 208, 379 (1983).

[17] M. Mundschau, Synchr. Rad. News 4, 29 (1991).

[18] B. Lai, F. Cerrina, J. Underwood, SPIE 563, 174 (1985).

[19] J. Welnak, Z. Dong, H. Solak, J. Wallace, F. Cerrina, M. Bertolo, A. Bianco, S. DiFonzo, S. Fontana, W. Jark, F. Mazzolini, R. Rosei, A. Savoia, J.H. Underwood, G. Margaritondo, Rev. Sci. Instrum. 66, 2273 (1995).

[20] F. Gozzo, M. Marsi, H. Berger, G. Margaritondo, A. Ottolenghi, A.K. Ray-Chaudhuri, W. Ng, S. Liang, S. Singh, J.T. Welnak, J.P. Wallace, C. Capasso, F. Cerrina, Phys. Rev. B 48, 1763 (1993).

[21] F. Gozzo, H. Berger, I.R. Collins, G. Margaritondo, W. Ng, A.K. Ray-Chaudhuri, S. Liang, S. Singh, F. Cerrina, Phys. Rev. B 51, 5024 (1995).

[22] J. Kirz, C. Jacobsen, M. Howells, Quart. Rev. Biophys. 28, I, 33 (1995) and references therein.

[23] A. Warwick, J. Delinger, B. Tonner, Internal ALS-LBNL report, 1994.

[24] P. Kirkpatrick, A.V. Baez, J. Opt. Soc. Am., 766 (1948).

[25] H. Padmore, Internal ALS-LBNL report, 1996. 